\title{
Design and Implementation for Applications That Provide Andong Culture and Tourism Information
}

\author{
Sanghyung Kim, Jooryeong Kim, Yiseul Kwon, Sungjin Jung, \\ Eunju Park and Hankyu Lim* \\ Department of Multimedia Engineering, Andong National University, \\ 1375 Gyungdong-ro, Andong-City, Gyeongsangbuk-Do, Republic of Korea \\ tkdel5212@naver.com,theoryh@naver.com,kiai94@naver.com, \\ sj3428@hanmail.net,eunju@anu.ac.kr,*hklim@anu.ac.kr
}

\begin{abstract}
While the widespread use of smart devices has led to the development of various applications in our daily lives, there are not many travel applications for an area called Andong. For this reason, we designed and implemented 'an application, which provides information on culture and tourism in Andong'. We implemented voice recognition function and GPS-based position information function in the application to provide travellers visiting Andong with accurate information about the area, and to improve user convenience for those who are not familiar with the device. It is believed that the application will provide travelers and citizens in Andong with accurate information and convenient access to the information, as well as more choices for application.
\end{abstract}

Keywords: Smartphone Application, GPS, Voice Recognition, Web, Web Application

\section{Introduction}

Many countries around the world are developing tourism industry and providing various kinds of tourism services to attract more travelers [1]. The reason people travel is to satisfy their needs. Travelers look for information, which is a medium linking means of transportation, things to see, tourist facilities, and tourism services, with which to solve problems they encounter while traveling. Different people need different information in terms of its nature and amount, and new information is required when one prepares or goes on a trip [2].

'Mobile Trend of 1H 2015' conducted by KT Economic Research Institute said the distribution rate of smartphones in Korea was $83.0 \%$ as of March 2015. 'Searching data and information (99.8\%)', 'Communication (99.5\%)' and 'Leisure activity (96.4\%)' accounted for large shares [3] of activities that people do with mobile devices like smartphones. The widespread use of mobile devices has led to the expansion of the mobile application market, which in turn leads to the development of various applications [4]. When it comes to travel, however, mobile applications are rarely used as a source of information. A '2015 Survey on Travel in Korea' conducted by the Ministry of Culture, Sports and Tourism said that most travelers, family or individual, refer to 'portal websites' for information. Figure 1 shows major sources of information for family trips [5]. We believe the reason for this is that there is not enough applications providing travel information, or that the information provided (if any) is not that useful.

* Corresponding Author 


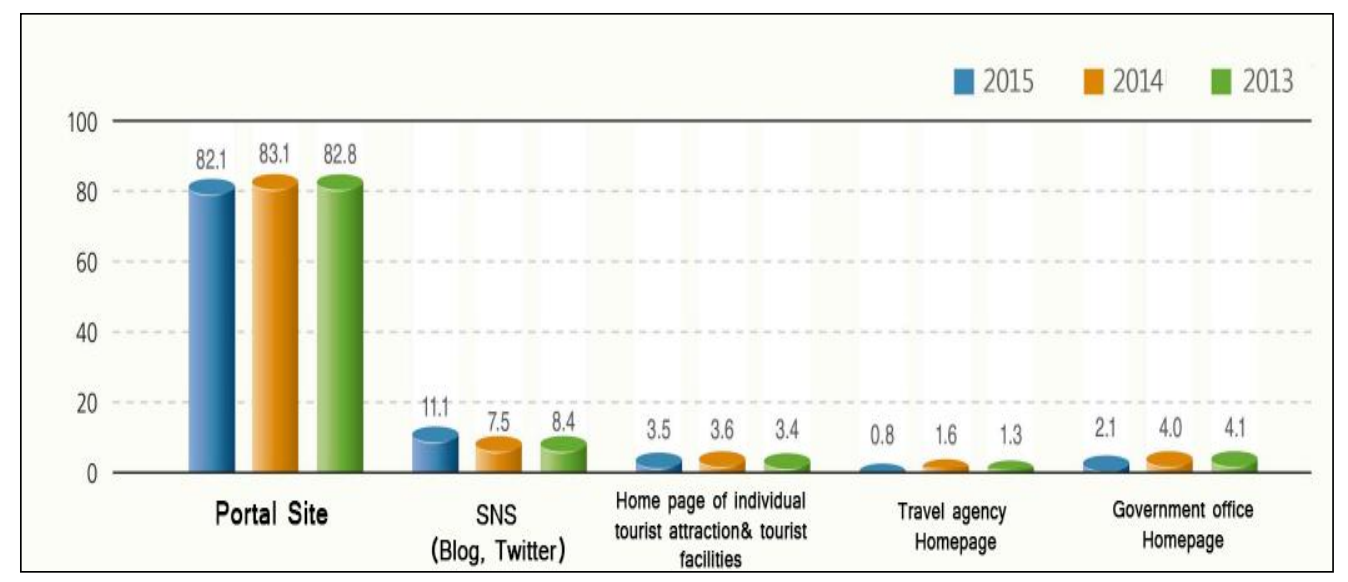

Figure 1. Main Internet Information Source when Traveling (measure: \%)

For Andong, there was one application called 'Andong stamp tour' providing travel information on the region. The application is run by the Andong festival tourism foundation. However, the usefulness of the application was rated below average as of Oct. 2016, with many negative reviews regarding errors in position recognition that caused users much inconvenience.

In this regard, we designed an application providing information on culture and tourism in Andong to provide people visiting the area, as well as citizens there, with information such as tourist attractions and famous restaurants. The application is operated through GPS and voice recognition functions, providing users with accurate information on their position and information about the place they want to go by showing an image on a map. In addition, a voice recognition function was added to the existing search functions to increase access for those who are not familiar with mobile devices. We believe that this application will be a source of tourist information for many smartphone users in Korea, while providing people visiting Andong with more application options.

\section{Related Research}

\subsection{GPS}

GPS recognizes the current location by analyzing electronic signals from four satellites. The distance between a GPS satellite and the measured location is obtained by comparing time information in electronic signals from a GPS satellite to the time when the information is received by a receiver, and then by calculating the moving distance of light according to time difference. If you measure distance from one satellite by using this, your current location is displayed on a spherical surface with a satellite as a center, as is shown in the very left side of Figure 2.

If you consider the distance measured from a second satellite, your current position is located in a circle, where spherical surfaces with two satellites as a center are met, as is shown in the middle of Figure 2. If you obtain the point of intersection of a circle measured by two satellites and the distance from a 3rd satellite, your position is located at two positions of a circle obtained by the very right side of Figure 2. Since one of the two positions is located in space or deep underground, both of which are inaccessible, the other becomes your position. Lastly, if you set a 4th satellite and time errors, and set the time of a receiver by that of a satellite to correct errors, you can find your accurate position [6].

GPS function allows users to find popular restaurants, tourist attractions, and accommodations in an easy and accurate way. We used Google GPS, an Open API, to 
help allow users to find location information that they want, as well as their current location [7].

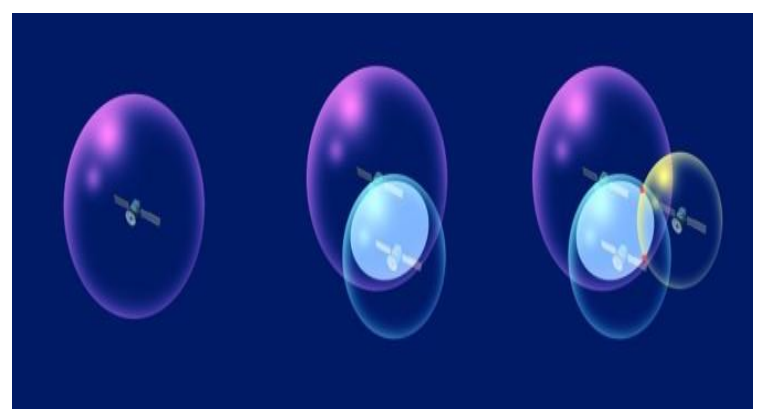

Figure 2. GPS Function

\subsection{Voice Recognition}

Voice recognition technology is about analyzing voice input, extracting its characteristics, measuring similarities with previously collected voice model database, and switching the most similar thing into character or command [8]. Voice recognition technology is a kind of pattern recognition process. Since people have different voices, pronunciations, and intonations, voice recognition technology collects voice data from as many people as possible to extract common characteristics and establish a pattern. Voice recognition process consists of a preconditioning process and recognition process as is shown in Figure 3 [8].

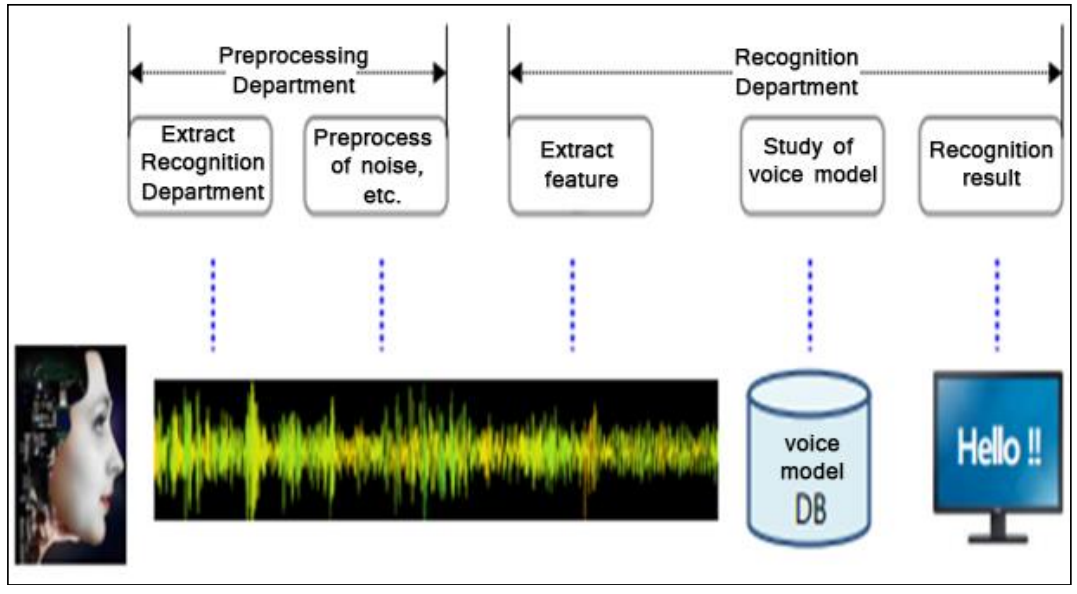

Figure 3. Fundamentals of Voice Recognition Technology

The section to be recognized is found from a user's voice; here, noise components are removed and characteristics are extracted through recognition process in the preprocessing area. The input voice is compared with a voice database to display the most possible results in the recognition area. Language model is used to limit words that are compared to increase recognition performance when a sentence, not a simple command, is recognized. Such a process emulated the cognitive process in which a person is born, standardizes numerous words and grammar in his/her head through a lot of repetition, and recognizes a new voice based on this [9]. In this paper, voice recognition function was used to search a location that a user wanted to go to, and then provide the search results through voice. 


\section{Design}

\subsection{Application Benchmarking}

'Pikicast' is a content platform that provides optimized content for web and mobile devices, which $60 \%$ of Korea's population in their teens and 20 s use. One of its advantages is that its registration process is simple. What's special about this platform is that users can sign in to the platform through Kakao Talk or Facebook, and that they can slide the screen by touching it and keep reading the contents without being distracted [10]. Figure 4 shows benchmarked 'Pikicast'.

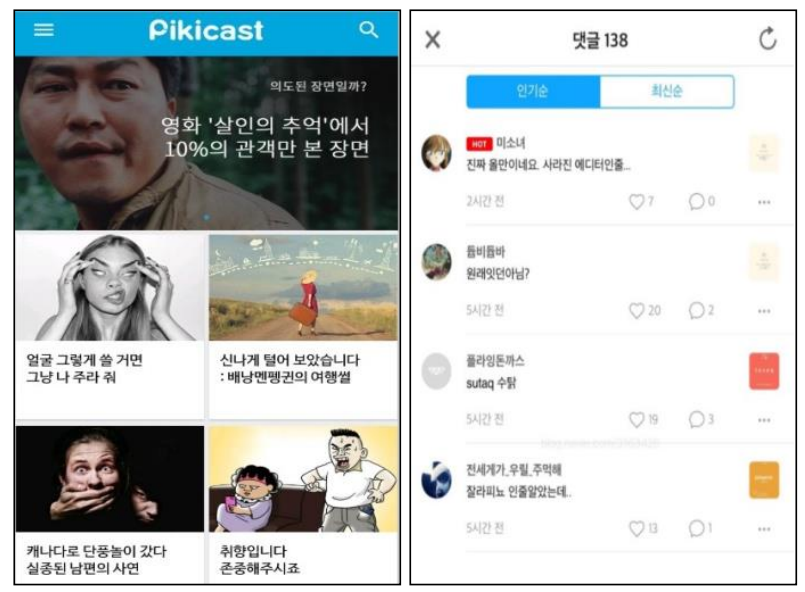

Figure 4. 'Pikicast'

We checker boarded famous restaurants and tourists attractions in Andong, as is shown in Figure 4 to decrease a sense of distance and provide information users with information in a convenient and friendly way.

The way contents are arranged in 'Pikicast' are user friendly; they are easy to read. However, as the amount of contents increased, so did the provocative ones. In addition, increasing the number of users may lead to increased malicious messages and disputes among users. In this regard, we implemented functions with which users can check various destinations and famous restaurants, without sign-in and comment functions. An application called 'Jejuwang' is a useful application that provides users with various information such as famous restaurants, tourist attractions, accommodations, and discount information. Users can share various information about a specific topic and check comments. In addition, users can make their own travel plan, and post it to share it with others, as is shown in Figure 5. Posts are uploaded either by an administrator or the users. For this reason, most comments are subjective, as is shown in the right side of Figure 5. This means people cannot solely depend on posts to plan their travel.

For this reason, we did not develop a comment function to prevent wrong information based on users' subjective opinions to ensure information is provided only by an administrator. 


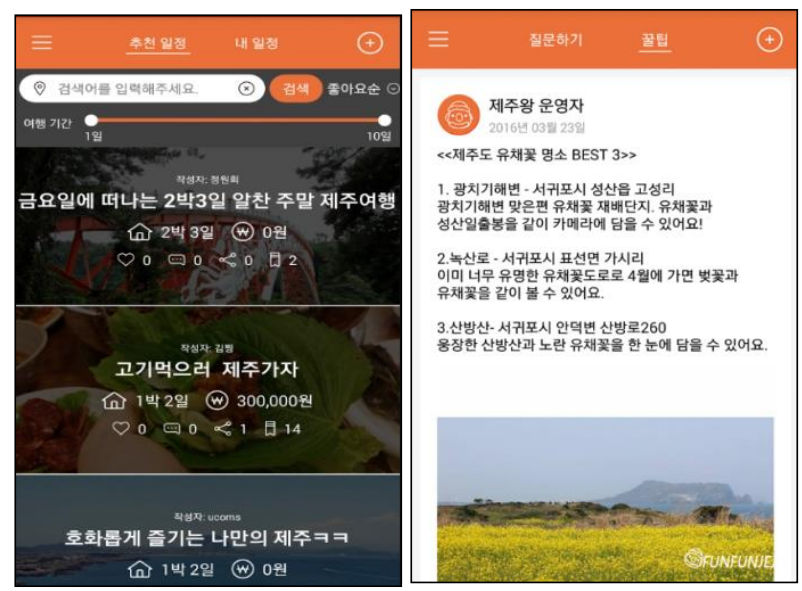

Figure 5. 'Jejuwang'

\subsection{System Structure Diagram}

Figure 6 shows the overall structure diagram of the application that we implemented. There are a total of 5 categories. Voice search is conducted and 'Tourism list' is listed on a main page. A 'Nearby tourist attraction' category shows nearby tourist attractions based on the current GPS location. 'Food' and 'Accommodation' categories are linked to famous restaurants and accommodations found in Andong.

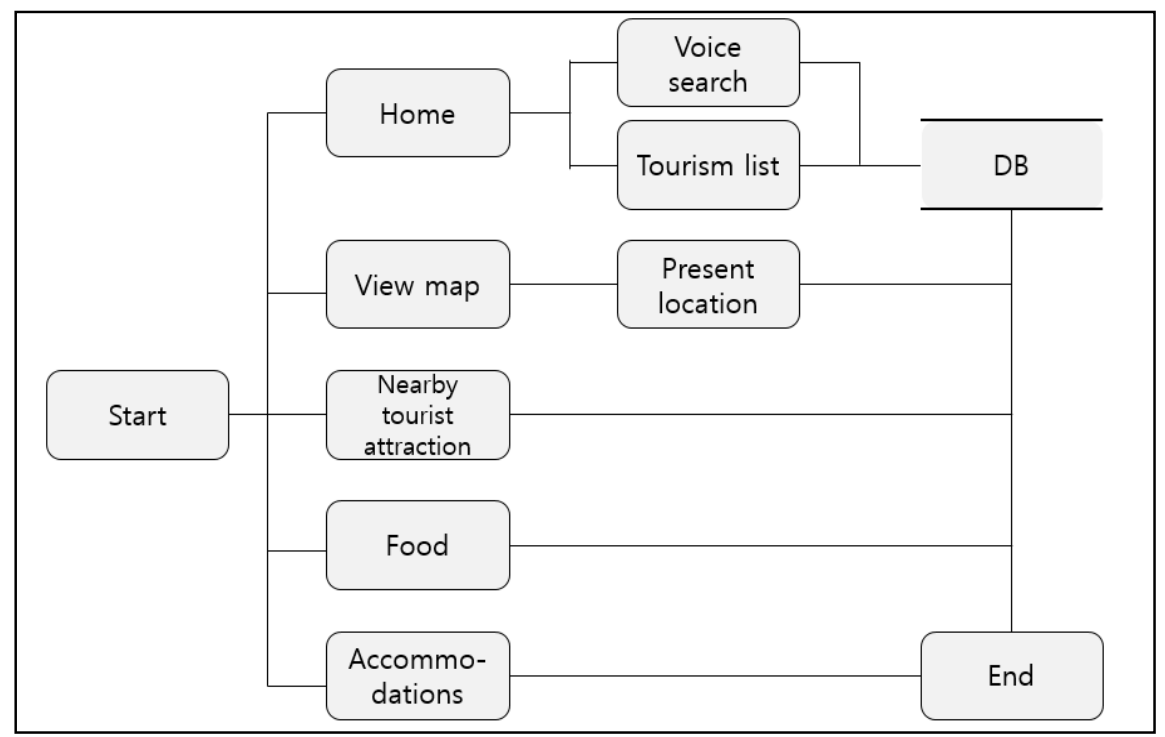

Figure 6. System Structure Diagram

\subsection{UI Design}

Figure 7 shows the 'UIs of a main screen' of the application. Only logos that show the characteristics of the application were inserted on the main screen, and the icons were designed based on the design of the 'Hahoe mask' representing Andong. 


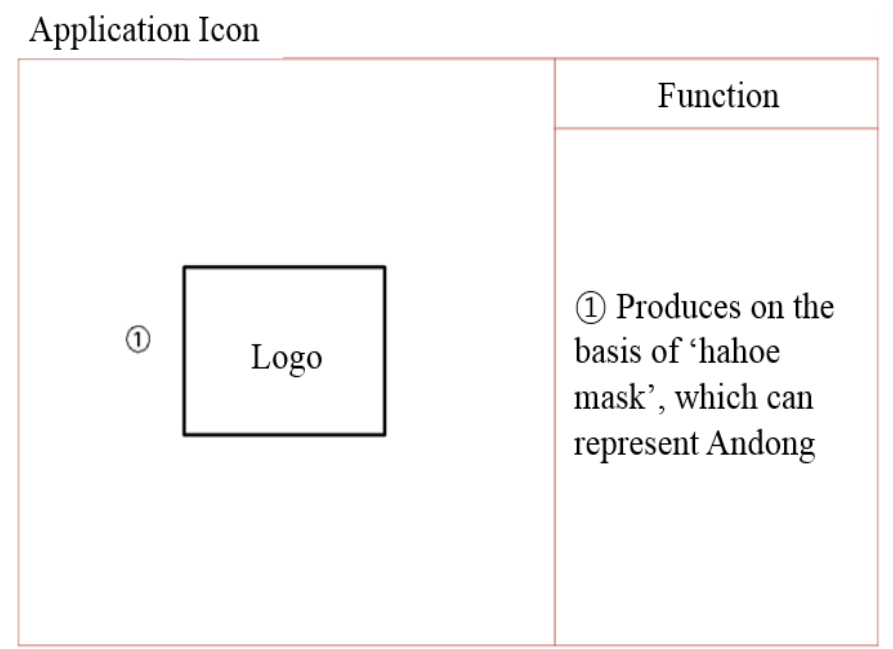

Figure 7. Main Screen UI

Figure 8 shows 'UIs on waiting screen', which appear when an application is run. Icon logo was inserted in the middle of the screen to indirectly provide users with the nature and mood of Andong, and the image of 'Dosanseowon Confucian Academy' was used as a background.

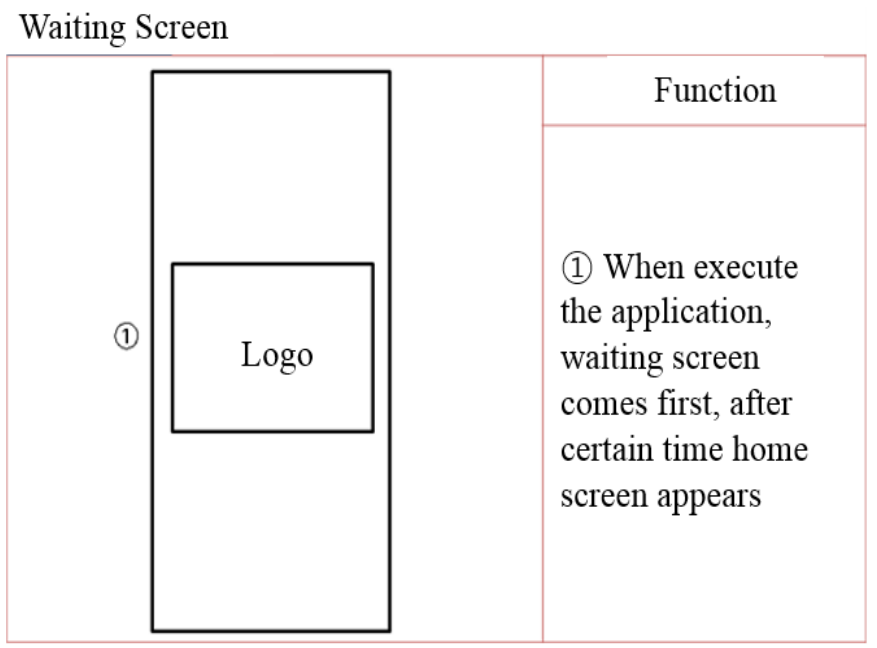

Figure 8. Waiting Screen UI

Figure 9 indicates 'UIs on home screen', where the Search window was located so users can find the tile of application and travel information on Andong. Travel contents were checker boarded in the middle of the screen to show all the information at once, and a simple image and descriptions are provided. In addition, the application was designed in a way to set the current location and allow users to choose the category they want. 


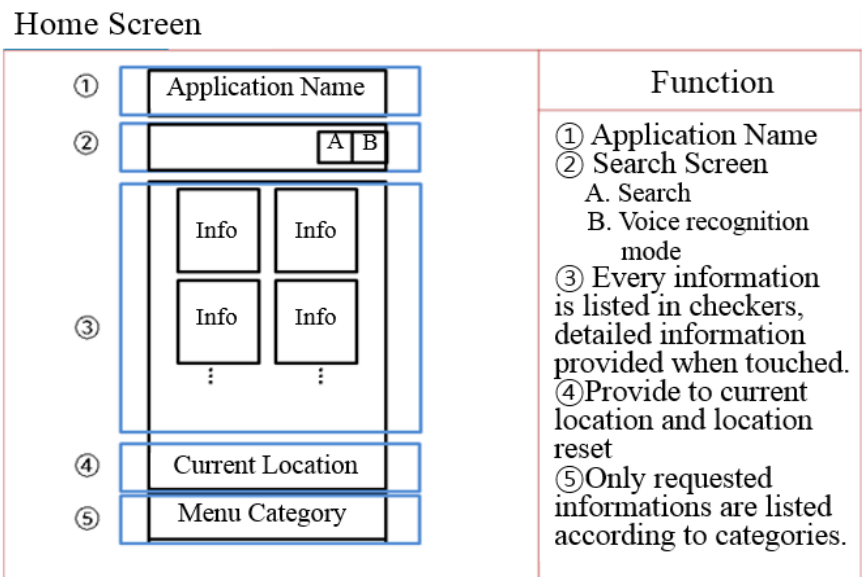

Figure 9. Home Screen UI

Figure 10 shows 'UIs on information screen', which is displayed after choosing either 'home screen', or category, and touching information appear in the middle of the screen. This screen shows the image, address, and description of the information, and in the bottom of the screen, GPS-based position information is displayed. The current location of a user and the information that a user is looking for are compared to help users easily find the location.

\begin{tabular}{|c|c|c|}
\hline (1) & Application Name & Function \\
\hline (2) & \begin{tabular}{|l|l|}
$\mathrm{A}$ & $\mathrm{B}$ \\
\end{tabular} & \multirow{6}{*}{$\begin{array}{l}\text { (1) Application Name } \\
\text { (2) Search Screen } \\
\text { A. Search } \\
\text { B. Voice recognition } \\
\text { mode } \\
\text { (3) Detailed description } \\
\text { of related information } \\
\text { (4)Provide to current } \\
\text { location and location } \\
\text { reset } \\
\text { (5) Only requested } \\
\text { informations are listed } \\
\text { according to categories. }\end{array}$} \\
\hline \multirow{3}{*}{ (3) } & Image of info & \\
\hline & $\begin{array}{l}\text { Name, Address, } \\
\text { Information }\end{array}$ & \\
\hline & $\begin{array}{l}\text { Location Info } \\
\text { according to GPS }\end{array}$ & \\
\hline (4) & Current Location & \\
\hline (5) & Menu Category & \\
\hline
\end{tabular}

\section{Figure 10. Information Screen UI}

\section{Implementation}

\subsection{STT (Speech to Text)}

In the study, Speech to Text, voice conversion technology was used to search information on tourist attractions and receive the searched information.

Figures 11 and 12 show where the SST was coded by using Android Studio tool. The Internet and recordable manifest are set to uses-permission value.

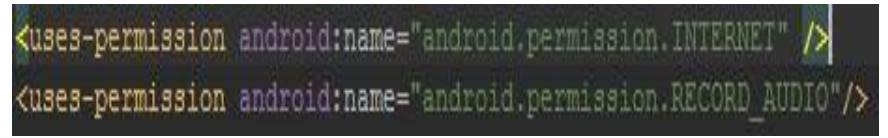

Figure 11. Voice Recognition Manifest Set Up 
As is shown in Figure 12, when a user inputs voice into application, it is stored in ArrayList $<$ String $>$ object, converted into texts through toArray() function.

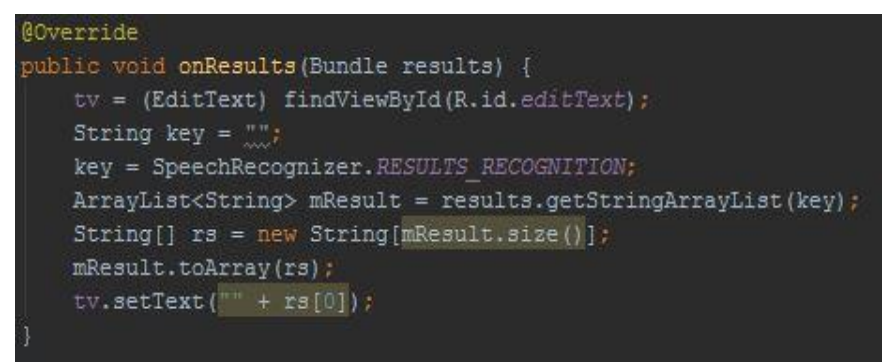

Figure 12. Speech to Text

\subsection{GPS}

GPS function links value stored in Google Map to display user his/her current location. Figure 13 shows a screen where manifest was set to find GPS value.

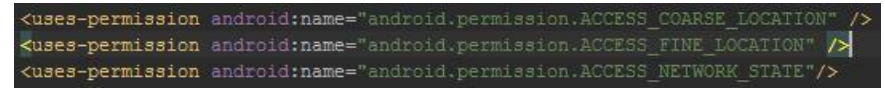

Figure 13. GPS manifest

Figure 14 shows code that imports current latitude and longitude from an object called GpsInfo.

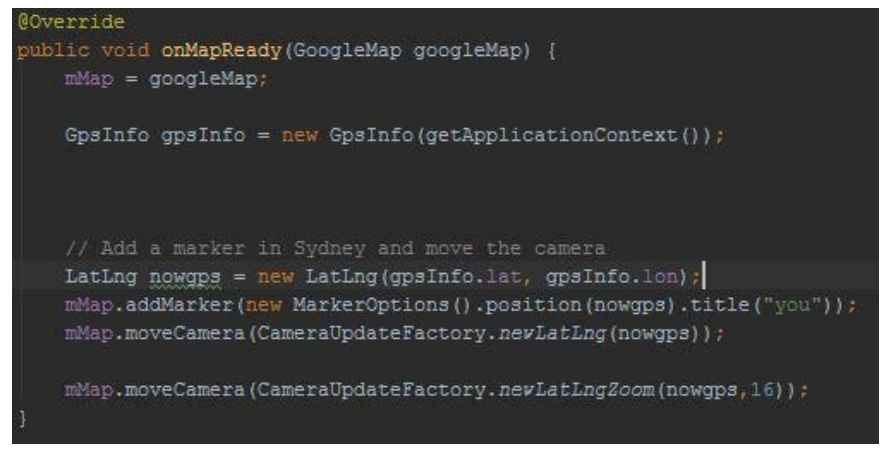

\section{Figure 14. Store the Figure of Present Location}

Figure 15 shows code that display contents of GPS after finding SupoortMapFragment by using a command, findFragmentById.

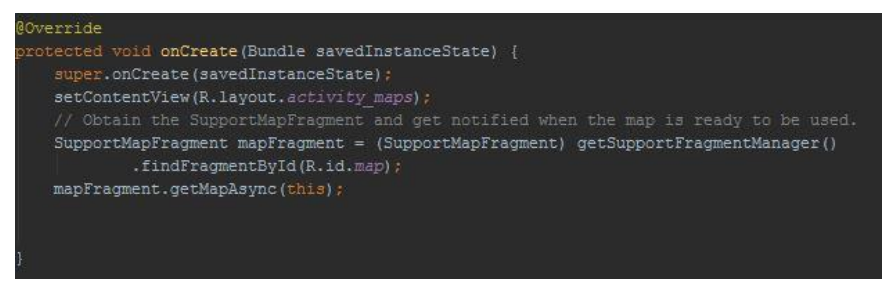

Figure 15. Print Out the GPS Map

\subsection{Implementation Image}

Figure 16 shows 'Icons of application' and 'Idle screen'. Adobe Photoshop CS6 and Adobe illustrator CS6 were used as manufacturing tools, and the icon was developed based on the design of the 'Hahoe mask', which represents Andong. In addition, the 
image of 'Dosanseowon Confucian Academy' was used for Idle screen to express the nature of Andong.
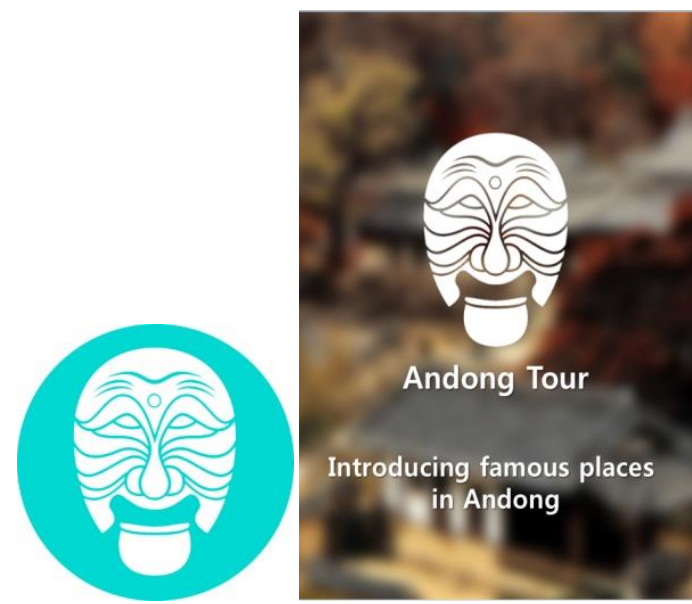

Figure 16. 'Icons of Application' and 'Idle screen'

Figure 17 shows 'Home screen' and 'Information screen' based on UI design. Users can search information on Andong by touching icons that look like a magnifying glass or a microphone on the search window of the 'Home screen'. Categorical image and short descriptions are displayed in the middle of the screen, and users can see the image and description by scrolling the screen up and down. Menu category is displayed by scrolling the screen from side to side to increase user concentration.

Relative distance is displayed by calculating distance between a user's current location and the location of a tourist attraction that user wants to go to by using 'Set My Location' function in the bottom of the screen. The distance between the image and me, name, the category, address, and information are displayed in 'Information screen', and GPS-based information is displayed as an image at the bottom of the screen.

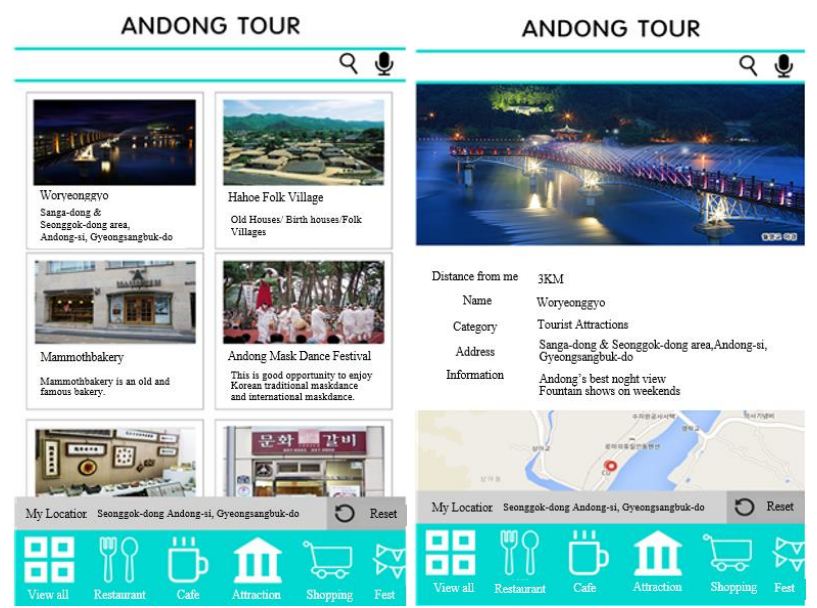

Figure 17. 'Home Screen' and 'Information Screen'

Figure 18 shows a screen where a user's current location is displayed by using GPS. 


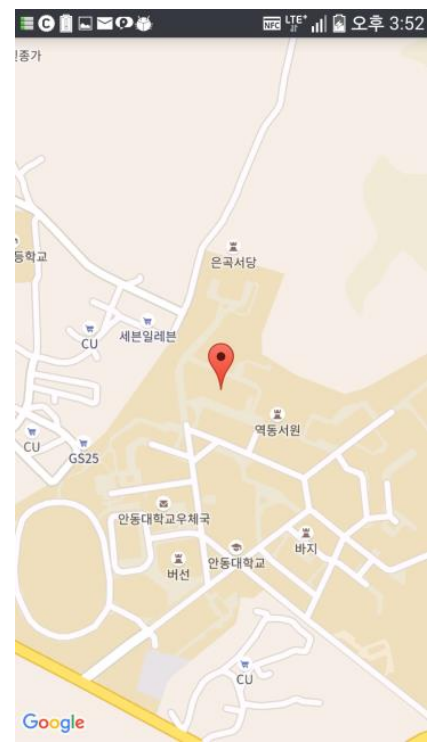

Figure 18. Show Current Location using GPS

\section{Conclusion}

The increased use of smartphones has led to the development of various kinds of applications, however, there are not many applications providing information on Andong. In this regard, we designed and implemented an application that provides information on culture and tourism in Andong. The application implemented in this study used location information and voice recognition function to allow travelers visiting Andong to have access to accurate information and to increase user convenience. We believe this will provide travelers visiting Andong and its citizens with information on the area and more options for applications by alternating the existing ones, which lacked information and were hard to implement.

People go on a trip with their family, friends, or by themselves to get out of their daily routines, create new experiences, make good memories, and even refresh. Getting information on the place they want to go to before going on to trip will provide them high-quality travel experience, and we believe the application implemented in this study will be of great help.

\section{Acknowledgments}

This work was supported by a grant from 2016 Seoul Accord Project (R0613-16-1148) of MISP (Ministry of Science, ICT and Future Planning) and IITP (Institute for Information and Communication Technology Promotion).

\section{References}

[1] Ministry of Strategy and Finance, "Trends in cultivating service industries in major countries and policy implication", Ministry of Strategy and Finance, (2008).

[2] J.-S. Kim, “A Study on the Traveler's Type for the Tourism Information Service”, Graduate School of Techno Design from Kookmin University, (2010).

[3] KT Economic Research Institute, "Mobile Trend of 1H 2015", KT Economic Research Institute, (2015).

[4] B. Jung, "Current status and prospects of mobile application market", KISDISTAT REPORT, vol 26, no 1, (2014).

[5] Ministry of Culture, "2015 Survey on Travel in Korea", Ministry of Culture, (2015).

[6] http://terms.naver.com/entry.nhn?docId=1051240\&cid=47341\&categoryId=47341

[7] G. Lee, U. Yun, G. Pyun, H. Ryang, "Design and Implementation of Application for Requesting Help using GPS Information in Android Platform”, Korean Society For Internet Information, (2012), pp.7374. 
[8] G. Lee, "Artificial intelligence-based speech recognition, better than a person”, Digieco Issues \& Trend, (2016).

[9] S.-M. Lee, "Study on the limitation factors of speech recognition technology in mobile", Graduate School of Advertisingand Public Relations of Hongik Univesity, (2012), pp. 14-15.

[10] http://blog.naver.com/mintkiwi24/220302800654.

\section{Authors}

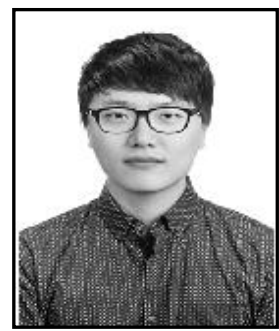

Sanghyung Kim took up Multimedia Engineering from Andong National University in 2011. He currently is a student of Multimedia Engineering from Andong National University.

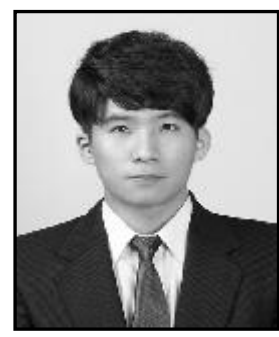

Jooryeong Kim took up Multimedia Engineering from Andong National University in 2011. He currently is a student of Multimedia Engineering from Andong National University.

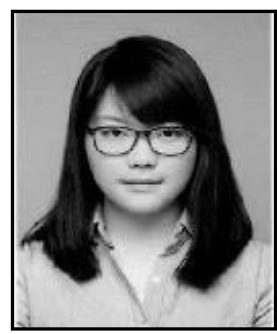

Yiseul Kwon took up Multimedia Engineering from Andong National University in 2013. She currently is a student of Multimedia Engineering from Andong National University.

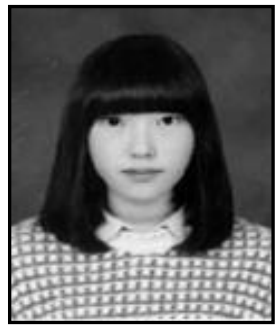

Sungjin Jung took up Multimedia Engineering from Andong National University in 2013. She currently is a student of Multimedia Engineering from Andong National University.

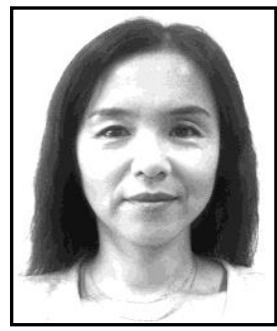

EunJu Park received her B.S. degree in Computational Statistics from Andong National University in 1993. She received her M.S. degree in Computer Engineering from Andong National University in 2001. She received her $\mathrm{Ph}$. D. degree in Information Communication Engineering from Andong National University in 2015. She currently is a full-time lecturer at Andong National University. 


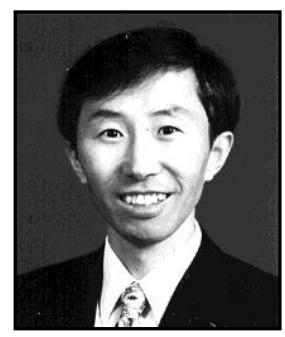

Hankyu Lim received his B.S. degree in Electronics Engineering from Kyungbook National University in 1981. He received his M.S. degree in Computer Engineering from Yonsei University in 1984. He received his PH. D. degree in Computer Engineering from Sung Kyun Kwan University in 1997. He is currently a professor at Andong National University. 